\title{
$O$-Acetylation of cellulose and monosaccharides using a zinc based ionic liquid $\dagger$
}

\author{
Andrew P. Abbott, ${ }^{a}$ Thomas J. Bell, ${ }^{a}$ Sandeep Handa*a ${ }^{* a}$ and Barry Stoddart ${ }^{b}$ \\ Received 16th August 2005, Accepted 18th August 2005 \\ First published as an Advance Article on the web 5th September 2005 \\ DOI: 10.1039/b511691k
}

The efficient $O$-acetylation of monosaccharides and cellulose is demonstrated using a Lewis acidic ionic liquid based on choline chloride and zinc chloride.

\section{Introduction}

The application of ionic liquids as environmentally benign reaction solvents for synthesis and catalysis has received extensive recent attention. ${ }^{1-4}$ In this regard we have developed ionic liquids based on the cheap and readily available components choline chloride $\left(\mathrm{ChCl} ; \mathrm{HOCH}_{2} \mathrm{CH}_{2} \mathrm{~N}(\mathrm{Me})_{3} \cdot \mathrm{Cl}\right)$ and zinc chloride as alternatives to the more commonly employed alkyl imidazolium-aluminium chloride mixtures. ${ }^{5}$ In an analogous manner to the chloroaluminate systems, we have shown that these zinc based liquids form complex anions of the form $\mathrm{ZnCl}_{3}{ }^{-}$and $\mathrm{Zn}_{2} \mathrm{Cl}_{5}{ }^{-}$. Although these species are less Lewis acidic than their aluminium counterparts the $\mathrm{ChCl}-\mathrm{ZnCl}_{2}$ ionic liquids are water insensitive and zinc is environmentally more benign. These Lewis acidic solvents have been successfully employed for a variety of reactions including Diels-Alder, Fisher indole synthesis and polymerisations. ${ }^{6,7}$ In this current study we report that $O$-acetylation of sugars and cellulose can effectively be carried out using chlorozincate ionic liquids.

The $O$-acetylation of carbohydrates is widely employed not only for the protection of hydroxy groups but also for the purification and structural elucidation of natural products. Per- $O$-acetylation is most frequently achieved using excess acetic anhydride together with a wide variety of catalysts including, amongst others, pyridine $^{8}$ (and its derivatives), ${ }^{9}$ sodium acetate, ${ }^{10} \mathrm{FeCl}_{3},{ }^{11}$ metal triflates, ${ }^{12}$ and $\mathrm{ZnCl}_{2}{ }^{10}$ The reaction has also been carried out in ionic liquids although only imidazolium based salts have been employed to date. Thus, the acetylation of carbohydrates has been demonstrated in imidazolium systems containing dicyanamide ${ }^{13}$ or benzoate ${ }^{14}$ anions and in both cases the ionic liquid acted as both solvent and catalyst for the reaction. In addition ionic liquids have also recently been employed as media for the enzymatic acylation of glycosides. ${ }^{15}$

\footnotetext{
${ }^{a}$ Department of Chemistry, University of Leicester, Leicester, UK LE17RH.E-mail: s.handa@le.ac.uk; Fax:+44 (0)116 252 3789; Tel: $+44(0) 1162522128$

${ }^{b} P \& G$ Technical Centres, Whitley Road, Longbenton,

Newcastle Upon Tyne, UK NE12 9TS

$\uparrow$ Electronic supplementary information (ESI) available: Experimental procedures and IR calibration plot. See http://dx.doi.org/10.1039/ b511691k
}

\section{Results and discussion}

To test the efficacy of Lewis acidic ChCl-based mixtures for the acetylation of carbohydrates the compounds $\mathbf{1}-\mathbf{4}$ were reacted with acetic anhydride in the ionic liquid for $3 \mathrm{~h}$ at $90{ }^{\circ} \mathrm{C}$ (Scheme 1). $\dagger$ Substrates 1-4 were completely soluble under the reaction conditions and the mixtures were worked up by the addition of water followed by extraction with ethyl acetate to give in each case only the per- $O$-acetylated product $\mathbf{1 a}-\mathbf{4 a}$. Table 1 summarises the results of these reactions. It can be seen (entries 1-4) that the $[\mathrm{ChCl}]\left[\mathrm{ZnCl}_{2}\right]_{2}$ ionic liquid can be successfully employed as both solvent and catalyst for the acetylation of simple monosaccharides. The yields for these conversions are remarkable considering the potential side reaction involving acetylation of choline (present in ca. 20 mole equivalents to the substrate). In fact reaction of $\mathbf{1}$ using an acetylcholine chloride ( $\mathrm{AcChCl}$ )-zinc chloride mixture (entry 5) gave a similar yield of 1a suggesting that the potential side reaction does not significantly affect the process. The reaction is also successful with the less Lewis acidic $[\mathrm{ChCl}]\left[\mathrm{SnCl}_{2}\right]_{2}$ ionic liquid (entry 6) albeit with a slightly reduced yield. However, a eutectic mixture of $[\mathrm{ChCl}][\mathrm{Urea}]_{2}$ (which forms an ionic liquid analogue but has no Lewis acidity) ${ }^{16}$ gave no significant reaction (entry 7). In addition to acetylation the $[\mathrm{ChCl}]\left[\mathrm{ZnCl}_{2}\right]_{2}$ system also supported reaction of $\mathbf{1}$ with propionic and isobutyric anhydrides to give, under the same reaction conditions, only the corresponding per- $O$-acylated products in $65 \%$ and $33 \%$ yield respectively (unoptimised yields, data not shown).

The most intriguing result is from the reaction of $\mathbf{1}$ with only 1 equivalent of acetic anhydride (entry 8). The only product isolated from this reaction was $1 \mathbf{a}\left(96 \%\right.$ yield based on $\left.\mathrm{Ac}_{2} \mathrm{O}\right)$ with no evidence for any partially acetylated intermediates. This result compares favorably with our observations for the reaction of 1 with 1 equivalent of acetic anhydride under conventional conditions in either pyridine (giving a complex mixture of partially acetylated intermediates) or $\mathrm{ZnCl}_{2}$ in THF (giving predominately 1a together with significant amounts (ca. 20-30\%) of partially

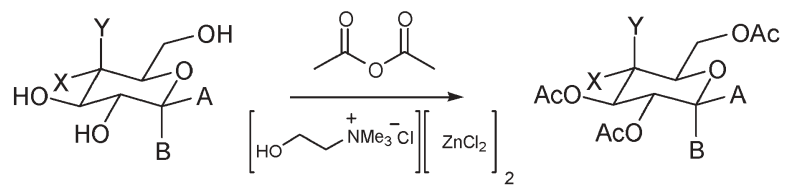

1: $\mathrm{X}=\mathrm{OH}, \mathrm{Y}=\mathrm{H} ; \mathrm{A}=\mathrm{H}, \mathrm{B}=\mathrm{OMe}$ 2: $\mathrm{X}=\mathrm{H}, \mathrm{Y}=\mathrm{OH} ; \mathrm{A}=\mathrm{H}, \mathrm{B}=\mathrm{OMe}$

3: $\mathrm{X}=\mathrm{H}, \mathrm{Y}=\mathrm{OH} ; \mathrm{A}=\mathrm{OMe}, \mathrm{B}=\mathrm{H}$

4: $\mathrm{X}=\mathrm{OH}, \mathrm{Y}=\mathrm{H} ; \mathrm{A}=\mathrm{H}, \mathrm{B}=\mathrm{OH}$ 1a: $X=O A c, Y=H ; A=H, B=O M e$ 2a: $X=H, Y=O A c ; A=H, B=O M e$ 3a: $X=\mathrm{H}, Y=O A c ; A=O M e, B=H$ 4a: $X=O A C, Y=H ; A, B=H, O A C$
Scheme 1 Acetylation of monosaccharides in ChCl-based ionic liquids. 
Table 1 Acetylation reactions of monosaccharides in ChCl-based ionic liquids at $90{ }^{\circ} \mathrm{C}$ for $3 \mathrm{~h}$

\begin{tabular}{|c|c|c|c|c|}
\hline Entry & Substrate & Solvent & $\mathrm{Ac}_{2} \mathrm{O}$ (eq.) & Yield $(\%)^{a}$ \\
\hline 1 & 1 & {$[\mathrm{ChCl}]\left[\mathrm{ZnCl}_{2}\right]_{2}$} & 5 & 78 \\
\hline 2 & 2 & {$[\mathrm{ChCl}]\left[\mathrm{ZnCl}_{2}\right]_{2}$} & 5 & 81 \\
\hline 3 & 3 & {$[\mathrm{ChCl}]\left[\mathrm{ZnCl}_{2}\right]_{2}$} & 5 & 78 \\
\hline 4 & 4 & {$[\mathrm{ChCl}]\left[\mathrm{ZnCl}_{2}\right]_{2}$} & 5 & $79^{b}$ \\
\hline 5 & 1 & {$[\mathrm{AcChCl}]\left[\mathrm{ZnCl}_{2}\right]_{2}$} & 5 & 66 \\
\hline 6 & 1 & {$[\mathrm{ChCl}]\left[\mathrm{SnCl}_{2}\right]_{2}$} & 5 & 68 \\
\hline 7 & 1 & {$[\mathrm{ChCl}][\text { urea }]_{2}$} & 5 & $<5$ \\
\hline 8 & 1 & {$[\mathrm{ChCl}]\left[\mathrm{ZnCl}_{2}\right]_{2}$} & 1 & $96^{c}$ \\
\hline
\end{tabular}

acetylated intermediates). The reason(s) for the selective formation of only 1a in the ionic liquid is not entirely clear. However, this novel finding is also observed in the reaction of $\mathbf{1}$ with 1 mole equivalent of propionic and isobutyric anhydrides (giving only per-acylated products in 88 and $68 \%$ respectively (based on anhydride)) and we believe that this may prove an effective method for producing per-acylated derivatives from synthetically or commercially valuable anhydrides.

In seeking to explore the potential environmental benefits of our system we next examined solvent recycling. Recovery of the solvent from the acetylation reactions was possible by employing a non-aqueous work up (involving simple extraction of the $[\mathrm{ChCl}]\left[\mathrm{ZnCl}_{2}\right]_{2}$ with ethyl acetate) followed by removal of any volatiles (presumably acetic acid) from the ionic liquid in vacuo. Pleasingly ionic liquid recovered in this way could be employed for the repeated acetylation of $\mathbf{1}$ without any reduction in the reaction yield (run $1-72 \%$, run $2-67 \%$, run $3-71 \%$, run $4-72 \%$ ). However, it should be noted that failure to remove volatiles from the recovered ionic liquid led to an appreciable decrease in the yields for subsquent reactions. Significantly the ionic liquid could also be re-used for different reactions with very low levels of cross contamination. Thus 4 was converted to $\mathbf{4 a}(75 \%, 3: 1$ mixture of $\alpha: \beta$ anomers) and the recovered ionic liquid was subsequently used for the acetylation of $\mathbf{1}$ to give the product $\mathbf{1 a}$ in good yield $(80 \%)$ and with $<1 \%$ (by ${ }^{1} \mathrm{H}$ NMR) contamination by $4 a$. This finding appreciably increases the potential scope for these ionic liquid reactions.

Having successfully demonstrated the acylation of monosacharrides we turned our attention to the acetylation of cellulose using the $[\mathrm{ChCl}]\left[\mathrm{ZnCl}_{2}\right]_{2}$ ionic liquid. Cellulose is traditionally acetylated using acetic anhydride, acetic acid and a sulfuric acid catalyst. The process gives a product in which all three $\mathrm{OH}$ groups are acetylated and which has relatively poor mechanical properties. Of more importance is the diacetate (generally termed cellulose acetate) which is obtained from the partial hydrolysis of the triacetate. Acetic anhydride and zinc chloride have also been used for the acetylation of cellulose, both neat and with a variety of solvents but this also gives exclusively the triacylated product. $^{17}$ Rogers and colleagues recently showed that cellulose was partially soluble in a variety of alkylimidazolium based ionic liquids and that those containing strong hydrogen bond acceptors $\left(\right.$ e.g. $\mathrm{Cl}^{-}$) were the most effective solvents especially when combined with microwave heating. ${ }^{18}$ Subsequent studies suggest that the polymer is disordered in these solutions. ${ }^{19}$ The first example of the acetylation of cellulose in an ionic liquid has recently been reported by Zhang and co-workers. ${ }^{20}$ The authors employed a novel allylimidazolium based ionic liquid and intriguingly the process was reported to occur under homogeneous conditions and in the absence of any catalyst. Cellulose acetates having a degree of substitution (DS) value of between 0.64-2.74 (corresponding to $21-91 \%$ acetylation) were produced using this system.

Using microwave heating as reported by Rogers we found that microcrystalline cellulose (ex. Sigma-Aldrich, DP ca. 200) was slightly soluble $(<3 \mathrm{wt} \%)$ in $[\mathrm{ChCl}]\left[\mathrm{ZnCl}_{2}\right]_{2}$. However, microwave conditions were not required for acetylation reactions which were carried out with $100 \mathrm{mg}$ of cellulose in $3 \mathrm{ml}$ of ionic liquid for $3 \mathrm{~h}$ at $90{ }^{\circ} \mathrm{C}$ (traditional heating) and with varying equivalents of acetic anhydride. At the end of the experiments water was added to the reaction mixture, precipitating the product which was centrifuged, filtered, washed with water and freeze dried. The resulting samples were initially analysed using infrared spectroscopy and the degree of acetylation estimated from the ratio of peak areas in the regions 3000-3500 (OH) and 1730-1750 (C=O) wavenumber and comparison to a calibration plot for different cellulose and cellulose acetate standard mixtures (see ESI $\dagger$ ). Table 2 shows a clear trend in that the degree of acetylation increases with the mole equivalents of acetic anhydride.

The functionalisation seen in these experiments could be due to per-acetylation of a fraction of the polymer chains or partial acetylation of all the chains. Since low molecular weight cellulose triacetate is soluble in acetone the samples were extracted with this solvent and only a small fraction was found to be soluble (ca. $1-10 \mathrm{wt}^{\%} \%$ ). IR analysis of this soluble material showed negligible absorbance at $3000-3500 \mathrm{~cm}^{-1}$ confirming it to be the triacetate and gel permeation chromatography (GPC) indicated a $M_{n}$ of between 2470-2580. We assume that this small amount of low molecular weight polymer is either present in the starting cellulose and/or is produced by glycoside hydrolysis under the reaction conditions. If the low molecular weight material is fully soluble in the ionic liquid then the per-acetylation is in accordance with the results seen with the monosacharides described above.

The vast majority $(>90 \mathrm{wt} \%$ ) of the material isolated from the acetylation reactions was insoluble in acetone. IR analysis of this insoluble fraction indicated that a significant proportion was acetylated and that the degree of acetylation increased with the equivalents of acetic anhydride used (Table 2). This finding raises the possibility of controlled synthesis of partially substituted cellulose acetates. The greater degree of functionalisation observed on increasing the equivalents of acetic anhydride is similar to the results reported by Zhang with their allylimidazolium based ionic liquid. ${ }^{20}$ However, it should be noted that these authors observed an unexplained drop in functionalisation when $>6.5$ equivalents of acetic anhydride were employed whereas the system reported here

Table 2 Acetylation of $\mathrm{OH}$ groups on cellulose with various mole equivalents of acetic anhydride in $[\mathrm{ChCl}]\left[\mathrm{ZnCl}_{2}\right]_{2}$ at $90{ }^{\circ} \mathrm{C}$ for $3 \mathrm{~h}$

\begin{tabular}{lcll}
\hline Entry & $\mathrm{Ac}_{2} \mathrm{O}(\mathrm{eq})$. & $\begin{array}{l}\text { Acetylated } \\
\text { groups (\%) }\end{array}$ & $\begin{array}{l}\text { Acetylated } \\
\text { groups (\%) }\end{array}$ \\
\hline 1 & 1 & 15 & 14 \\
2 & 7.5 & 17 & 14 \\
3 & 10 & 26 & 21 \\
4 & 20 & 47 & 31 \\
${ }^{a}$ Estimated by IR analysis. ${ }^{b}$ Analysis of total isolated material. \\
${ }^{c}$ Analysis of acetone insoluble fraction. \\
\hline
\end{tabular}




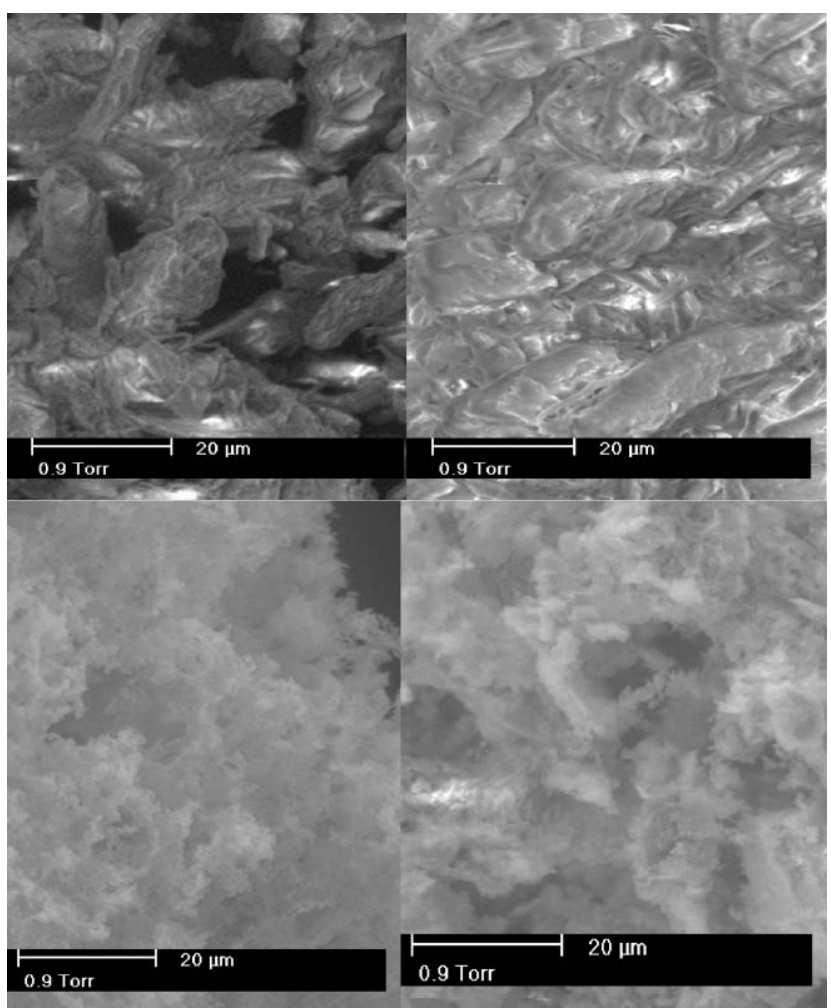

Fig. 1 SEM images of cellulose (top left), partially acetylated cellulose (top right), cellulose after dissolution in ionic liquid and regeneration with water (bottom left) and acetone soluble cellulose triacetate (bottom right).

shows increasing levels of derivatisation up to 20 equivalents of acetic anhydride used. Additionally, our $[\mathrm{ChCl}]\left[\mathrm{ZnCl}_{2}\right]_{2}$ system also allows access to cellulose acetate having low levels of functionalisation (i.e. $<20 \%$ acetylation).

Assuming that the hydroxyl groups can only react if they are wetted by the ionic liquid then this must involve significant restructuring of the polymer. Fig. 1 shows scanning electron microscopy (SEM) images of starting cellulose, partially acetylated cellulose, cellulose that has been dissolved in the ionic liquid and regenerated by the addition of water and low molecular weight cellulose triacetate. It can be seen that dissolution of the cellulose in the ionic liquid gives material having a homogeneous morphology which is consistent with the results of Rogers ${ }^{18}$ who found that the structure of cellulose was opened upon contact with the imidazolium based ionic liquids. The partially acetylated sample (top right) has a structure that is intermediary between crystalline starting cellulose and amorphous regenerated material suggesting that only a small fraction of the polymer chains have been opened up by the ionic liquid.

\section{Conclusion}

In summary this work has shown that ionic liquids based on $\mathrm{ZnCl}_{2}$ and $\mathrm{ChCl}$ are effective at carrying out $O$-acetylation reactions on a number of monosaccharides and cellulose. Per- $O$-acetylation is exclusively observed with monosacharrides and for low molecular weight polymer. Partial acetylation of cellulose is also possible with some control over the degree of modification.

\section{Acknowledgements}

The authors would like to acknowledge EPSRC (GR/R 39771), the Crystal Faraday Partnership and Procter and Gamble for funding this work.

\section{References}

1 P. Wasserscheid and T. Welton, Ionic Liquids in Synthesis, Wiley-VCH Verlag, Weinheim, Germany, 2003.

2 T. Welton, Coord. Chem. Rev., 2004, 248, 2459.

3 T. Welton, Chem. Rev., 1999, 99, 2071.

4 P. Wasserscheid and W. Keim, Angew. Chem. Int. Ed., 2000, 39, 3772.

5 A. P. Abbott, G. Capper, D. L. Davies and R. K. Rasheed, Inorg. Chem., 2004, 43, 3447.

6 A. P. Abbott, G. Capper, D. L. Davies, R. Rasheed and V. Tambyrajah, Green Chem., 2002, 4, 24.

7 R. C. Morales, V. Tambyrajah, P. R. Jenkins, D. L. Davies and A. P. Abbott, Chem. Commun., 2004, 158.

8 H. H. Schulubach and K. Repenning, Angew. Chem., 1959, 71, 193.

9 G. Hoefle and W. Steglich, Synthesis, 1972, 619.

10 A. I. Vogel, Vogel's Textbook of Practical Organic Chemistry, Wiley, New York, 5th edn, 1989, p. 644.

11 F. Dasgupta, P. P. Singh and H. C. Srivastave, Carbohydr. Res., 1980, 80, 346.

12 C-A. Tai, S. S. Kulkarni and S-C. Hung, J. Org. Chem., 2003, 68, 8719.

13 S. A. Forsyth, D. R. MacFarlane, R. J. Thomson and M. von Itzstein, Chem. Commun., 2002, 714.

14 S. Murugasen, N. Karst, T. Islam, J. M. Weincek and R. J. Linhardt, Synlett, 2003, 1283.

15 M-J. Kim, M. Y. Choi, J. K. Lee and Y. Ahn, J. Mol. Catal. B, 2003, 26, 115.

16 A. P. Abbott, G. Capper, D. L. Davies, R. Rasheed and V. Tambyrajah, Chem. Commun., 2003, 70.

17 B. Philipp, B. Lukanoff, H. Schleicher and W. Wagenknecht, Z. Chem., 1986, 26, 50.

18 R. P. Swatloski, S. K. Spear, J. D. Holbrey and R. D. Rogers, J. Am. Chem. Soc., 2002, 124, 4974.

19 J. S. Moulthrop, R. P. Swatloski, G. Moyna and R. D. Rogers, Chem. Commun., 2005, 1557.

20 J. Wu, J. Zhang, H. Zhang, J. He, Q. Ren and M. Guo, Biomacromolecules, 2004, 5, 266. 